\title{
Perceptual and response interactions in semantic priming
}

\author{
IRA H. BERNSTEIN, VICTOR BISSONNETTE, and KENNETH R. WELCH \\ University of Texas, Arlington, Texas
}

\begin{abstract}
In two experiments, subjects made pairs of lexical decisions verbally. In Experiment 1, masked stimuli appeared concurrently to the left and right of fixation; in Experiment 2, nonmasked stimuli appeared sequentially at fixation. The left-hand letter strings were judged more accurately in in Experiment 1, and the second letter strings were judged more accurately in Experiment 2. Each string in the pair could be either a word (e.g., fork) or a nonword anagram (e.g., frok). Consequently, the two strings in the pair could be related (e.g., fork-spoon, frok-spoon, etc.) or unrelated (e.g., fork-door, frok-door, etc.), independently of whether neither, either, or both strings were words. Semantically related stimuli induced consistent biases to respond "word," as noted in other studies. These biases were typically stronger for the event reported second. Minimal evidence was found for perceptual priming effects. The asymmetrical effects were consistent with spreading-activation-type mechanisms, but other considerations support a multiple-process view.
\end{abstract}

Priming involves the use of an accessory (prime) to influence a target's processing. In semantic priming, the potential influence is linguistic. The two events may be associates (Meyer \& Schvaneveldt, 1971; Neely, 1977) or belong to a common category, such as animal names (Battig \& Montague, 1969; Hines, Czerwinski, Sawyer, \& Dwyer, 1986). In other priming phenomena, the prime cues physical properties of the stimulus, such as spatial location (Lupker \& Massaro, 1979; Müller \& Findlay, 1987), which Farah (1989) terms perceptual priming.

Semantic priming (henceforth, simply priming) is typically concerned with the proactive ("forward") effects of the first stimulus on the second (or, if they are presented concurrently, the effects of the left-hand stimulus on the right-hand stimulus for normal English readers). Recent studies, however, also have been concerned with retroactive or "backward" priming (Briand, den Heyer, \& Dannebring, 1988; Stone \& Van Orden, 1989). Marcel's $(1980,1983 \mathrm{a}, 1983 \mathrm{~b})$ well-known studies have attempted to demonstrate "subliminal" priming. However, Bernstein, Bissonnette, Vyas, and Barclay (1989) noted that retroactive priming could explain Marcel's results as a reciprocal interaction between the ostensive prime and target. They showed that primes that were "subliminal" when presented alone were clearly "supraliminal" when presented with semantically related targets.

Priming has been obtained for naming latencies (Carr, McCauley, Sperber, \& Parmelee, 1982; Hines et al.,

A portion of these results was presented at the 1989 meeting of the Psychonomic Society in Atlanta, GA. The authors are grateful to Alison Foster, Linda Jerritts, Meeta Mangrola, Avni Vyas, and Dawn-Michelle Williams for their assistance in gathering data; to Thomas Wickens for his comments on categorical modeling; and to Robert Proctor and an anonymous reviewer for their insights. Requests for reprints should be addressed to Ira $\mathrm{H}$. Bernstein, Department of Psychology, University of Texas at Arlington, P.O. Box 19528, Arlington, TX 76019-0528.
1986), word/nonword (lexical) decisions (Antos, 1979; Meyer \& Schvaneveldt, 1971; Neely, 1977; O’Connor \& Forster, 1981; Schvaneveldt \& McDonald, 1981; Stone \& Van Orden, 1989), recognition memory (Ratcliff \& McKoon, 1988), and tachistoscopic accuracy (Bernstein et al., 1989). Briand et al. (1988) combined naming latencies and lexical decisions. Most often, interest centers on facilitation arising from a related prime (bread-butter), compared to either an unrelated prime (sleep-butter) or a nonword prime (selep-butter) (Antos, 1979; Bernstein et al., 1989; Schvaneveldt \& McDonald, 1981), but inhibition due to mispriming (bread-batter in a naming latency task) is also well documented (Norris, 1984). Neely (in press) provides an extensive empirical and theoretical summary of the priming literature.

In the present study, we are concerned with the extent to which prime/target interactions among "supraliminal" stimuli are symmetric. Some proposed mechanisms, such as spreading activation (Neely, 1977), readily handle proactive priming but have some difficulty in accounting for retroactive priming. Neely (in press) shows how spreading activation may be modified to account for retroactive priming in lexical decision via a feedback mechanism. However, he explicitly rejects this possibility on the basis of other data-namely, the difficulty in explaining the lack of retroactive priming in naming tasks. This led him to a multiprocess view of priming. Others, such as Ratcliff and McKoon's (1988) view that the ostensive prime and target form a compound cue, predict a more nearly symmetrical outcome.

A second issue is whether semantic priming involves a change in bias, sensitivity, or both. Farah (1989) has suggested that priming effects are criterion shifts (words and nonwords appearing more "wordlike"), unlike the sensitivity changes (word/nonword discriminations being made more efficiently) that are typical of perceptual prim- 
ing. She concluded that different theoretical mechanisms are needed to explain perceptual and semantic priming, despite attempts to unify them (Neely, 1977).

Schvaneveldt and McDonald (1981) found sensitivity changes in their latency measures but only bias changes in tachistoscopic accuracy, and they proposed a dual mode interpretation of priming. A holistic analysis of word shape occurs first, in which semantic relatedness can lower the criterion for a process like logogen activation (Morton, 1969), shifting criteria along Farah's (1989) lines. A "second look" then resolves ambiguities about the stimulus and may improve discrimination accuracy. Bernstein et al. (1989) suggested a similar mechanism.

Our approach to both issues was to present two letter strings $(A$ and $B$ ). Each could independently be a word or nonword formed by transposing two letters of a word. The $A$ and $B$ energies were equal, to allow the greatest opportunity for reciprocity. Subjects made conventional lexical decisions as to whether each string was a word or a nonword. The strings could be semantically related or unrelated; nonwords were considered related if they were derived from related words. Table 1 illustrates the resulting eight stimulus combinations and four response combinations.

The stimuli form a $2 \times 2 \times 2$ design: Each stimulus $(A$ and $B$ ) has two levels-word versus nonword - and semantic relatedness (which we will simply refer to as relatedness) also has two levels-related versus unrelated. Similarly, the response combinations form a $2 \times 2$ structure: Each response ( $a$ and $b$ ) has two levels, "word" versus "nonword." All main effects produce interactions. ${ }^{2}$ Specifically, the $a \times b$ interaction describes whether the two responses are the same or different. Also, $A$ is the target for its associated (proper) response $(a)$ and a prime for the other (improper) response $(b)$, and vice versa for $B$.

The four response probabilities within each row in $\mathrm{Ta}$ ble 1 add to 1.0. Thus, only three of the four response combinations within each row are free. The 32 cells of the table therefore contain $24 d f$. Three degrees of freedom estimate marginal response bias terms for $a, b$, and same versus different. The remaining 21 degrees of free- dom estimate the seven main effects on and interactions with each of these three responses' terms.

Our theoretical framework reflects Garner and Morton's (1969) concept of perceptual independence, which deals with how decisions interact, and Garner, Hake, and Eriksen's (1956) related consideration of converging operations, with which they attempted to separate perceptual and response systems. Both Ashby and Townsend (1986) and Wickens and Olzak (1989; Olzak \& Wickens, 1983; Wickens, 1989) have extended Garner and Morton.

We follow Wickens and Olzak's (1989) extension, and Figure 1 is similar to their Figure 1, adapted to priming. One effect, response bias-the preference for certain response categories over others-is not illustrated. $D e-$ tection (direct contingencies in Garner \& Morton, 1969) denotes the influence of stimuli on their proper responses (1A and $1 \mathrm{~B}$ in Figure 1). Sensory interference (crosscontingencies in Garner and Morton) refers to the influence of a stimulus on the other (improper) response (2A and 2B). Wickens and Olzak (1989) note in discussion that interference may be facilitative or inhibitory. Ashby and Townsend's (1986) term for the lack of such influence is perceptual separability. Wickens and Olzak (1989) discuss correlated sensory noise, bivariate numerical biases, and higher order associations, which fall under Garner and Morton's (1969) collective heading of response contingencies. These cannot be separated with our dichotomous ratings.

One can separate contingent detections (our term for perceptual interactions, identified as 3 in the figure) from contingent response biases (our term for decisional interactions, identified as 4), however. Contingent detections, like the $A \times$ relatedness interaction with $a$, involve a stimulus term, its proper response, and at least one other term. Corresponding contingent response biases, like the main effect of relatedness on $a$, do not include the proper stimulus.

Wickens and Olzak (1989) used hierarchical log-linear analysis, a form of categorical modeling. We begin by assuming that the four response frequencies are equal (.25) within each of the eight stimulus combinations. If this

Table 1

Row Conditional Response Probabilities within Conditions (Correct Response Pairings in Italics)

\begin{tabular}{|c|c|c|c|c|c|c|c|c|c|}
\hline \multirow[b]{3}{*}{ Stimulus } & \multirow[b]{3}{*}{ Example } & \multicolumn{8}{|c|}{ Experiment } \\
\hline & & \multicolumn{4}{|c|}{ Simultaneous/Masked } & \multicolumn{4}{|c|}{ Sequential/Unmasked } \\
\hline & & "WW" & "WN"' & "NW" & "NN"' & "WW" & "WN" & "NW" & "NN" \\
\hline RWW & fork-spoon & .69 & .18 & .10 & .03 & .92 & .01 & .06 & .00 \\
\hline $\mathbf{R W N}$ & apple-oraneg & .38 & .46 & .05 & .11 & .11 & .84 & .02 & .03 \\
\hline RNW & knief-gun & .27 & .08 & .46 & .19 & .18 & .01 & .79 & .02 \\
\hline RNN & sugra-ceram & .10 & .19 & .21 & .50 & .05 & .30 & .11 & .53 \\
\hline UWW & milk-door & .61 & .28 & .09 & .02 & .89 & .08 & .03 & .00 \\
\hline UWN & gold-tarin & .20 & .56 & .05 & .19 & .02 & .94 & .00 & .04 \\
\hline UNW & trukc-bird & .12 & .10 & .42 & .36 & .15 & .01 & .80 & .04 \\
\hline UNN & bxo-blal & .10 & .19 & .26 & .44 & .02 & .26 & .06 & .66 \\
\hline
\end{tabular}

Note-The first letter denotes level of semantic relatedness $(R=$ related and $U=$ unrelated $)$, the second letter denotes level of $A(\mathbf{W}=$ word and $N=$ nonword), and the third letter denotes level of $B$ (W $=$ word and $\mathbf{N}=$ nonword). 


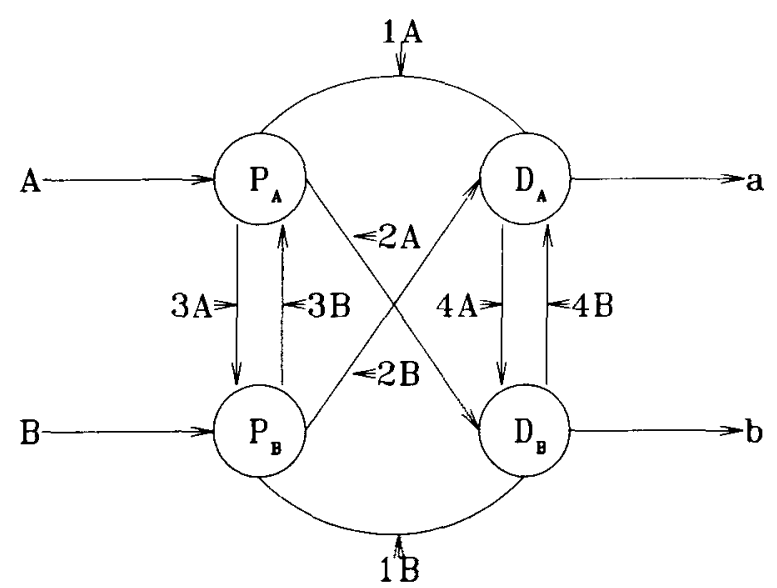

Figure 1. Possible interactions between Stimuli $A$ and $B$ and Responses $a$ and $b$ in a priming task. Processes $P_{A}$ and $D_{A}$ are the respective perceptional and decisional processes that mediate the relation between $A$ and $a$, and processes $P_{B}$ and $D_{B}$ are the respective perceptional and decisional processes that mediate the relation between $B$ and $b$. Effects $1 A$ and $1 B$ are detections (direct contingencies); Effects $2 A$ and $2 B$ are cross contingencies; Effects $3 A$ and $3 B$ are contingent detections, and $4 A$ and $4 B$ are contingent biases.

homogeneity model can be rejected, marginal response biases are estimated. Assuming further variance remains, detection parameters are estimated next. These collectively describe an independence model for a subject who judges $A$ and $B$ separately; residual effects reflect interdependent processing such as priming.

Detections are evaluated after biases because detections are more complex, by definition involving an additional stimulus term. Likewise, detections are evaluated before contingent effects, and contingent biases are evaluated before corresponding contingent detections because biases also involve one less term. For example, the bias for $a$ is derived from the marginal distribution of "word" versus "nonword" responses to $A$, but the detection of $A$ involves the two-way contingency between word versus nonword stimuli and "word" versus "nonword" responses. In general, one uses a hierarchical principle to account for lower order terms before higher order terms (cf. Bishop, Fienberg, \& Holland, 1975; Wickens, 1989). Contingent biases and detections involving relatedness are of the greatest interest.

Here, we will present data from two experiments in which different stimulus configurations were used. No direct comparison between them was intended; we simply wished to sample two of the many possible configurations that one might legitimately employ to establish the generality of relevant effects. In addition, we ran two control conditions in Experiment 1, which was pared down to one control condition in Experiment 2, to evaluate performance parity or relative performance under divided versus undivided attention (Garner \& Morton, 1969).

\section{EXPERIMENT 1}

The stimulus configuration in Experiment 1 is termed simultaneous/masked; $A$ and $B$ are respectively presented concurrently to the left and right of fixation and immediately pattern-masked.

\section{Method \\ Subjects. Fifty introductory psychology students participated in} partial fulfillment of course requirements. All were native English speakers with normal or corrected-to-normal vision.

Stimuli and Apparatus. Three separate stimulus lists each contained 120 word stimuli and 120 nonword stimuli. The word stimuli were common nouns with three to six letters, such as "WATCH." The nonword stimuli were common nouns with three to six letters, in each of which a pair of adjacent letters had been switched. For example, the word "WATCH" became the nonword "WACTH" through the switching of " $T$ " ' and " $C$." No nouns were used more than once on the stimulus lists. The subjects were given a spelling test at the end of the session, to ensure that they could spell the stimuli correctly.

The stimuli, which appeared in uppercase, were presented in the 40-column mode of a 12-in. Zenith Data Systems green monochrome monitor controlled by an Apple II + microcomputer. Each letter subtended $18^{\prime}$ at a $114-\mathrm{cm}$ viewing distance. The luminance of the green letters on the black background was $50 \mathrm{~cd} / \mathrm{m}^{2}$. Diener and Smee's (1984) algorithm was used to ensure presentation timing accuracy.

Procedure. The subjects were given a brief description of the experimental procedure in the laboratory. The ambient illumination was reduced to 35 lux, and the subjects were dark-adapted for $5 \mathrm{~min}$. They then received three sets of trials. The experimental trials consisted of 80 pairs of lexical decisions. The two sets of 40 control trials involved only one lexical decision. The target's location was unknown prior to its presentation in the uncued control condition, but it was known in the cued control condition. The presentation order of conditions and trials within conditions was randomized for each subject. Four practice trials preceded each of the three sets of trials.

Experimental trials. The subjects were exposed to: (1) a 900-msec fixation cross $(t)$ presented at the center of the screen, (2) a 500 -msec blank screen, (3) $A$ and $B$ appearing to the left and right of fixation together for $250 \mathrm{msec}$, and (4) two 100 -msec, 10-letter masks formed from the "QZQZQZQZQZ." Despite the constant exposure duration, no subject's performance was perfect. The $A$ stimulus appeared with its last letter one space to the left of fixation, and the $B$ stimulus appeared with its first letter one space to the right of fixation. Thus, two five-letter stimuli occupied a total of 13 spaces $\left(2.34^{\circ}\right)$. The subjects then verbally judged whether $A$ was a word or a nonword and then whether $B$ was a word or a nonword. The nouns were semantically related (e.g., CHAIR-DESK) on half of the trials and semantically unrelated (e.g., MILK-DOOR) on the remaining trials. A nonword was considered related to another stimulus if the word that produced it was related. Thus, CAHIR-DSEK are related nonwords because CAHIR is derived from CHAIR, DSEK is derived from DESK, and CHAIR and DESK are related. ${ }^{3}$

Uncued control trials. Trials in the uncued control condition were similar to the experimental trials, except that subjects made only one lexical decision. Following the fixation cross, two stimuli were presented, but only one was a lexical stimulus (word vs. nonword); the other stimulus was the character string “*****." After the fixation-stimuli-masks sequence, the subjects decided whether the left stimulus was a word, nonword, or "stars." This was repeated for 
the right stimulus. On half of the trials, the lexical stimulus was presented on the left; on the other half, it was presented on the right. The lexical stimulus was a word on half the trials and a nonword on half the trials.

Cued control trials. Cued control condition trials were identical to uncued control condition trials, except that the fixation stimulus was the letter " $L$ " (left) or " $R$ "' (right), which cued the lexical stimulus' side.

\section{Results}

Experimental condition. Table 1 contains the four response probabilities conditionalized on each of the eight stimulus combinations. The data are pooled over subjects, and thus ignore individual differences, which will be discussed below. In general, it can be seen that the largest probabilities for the response combinations are associated with their proper stimulus combination. For example, subjects said "word"'-"word" to a related word-word sequence .69 of the time. However, it is clear that the data must be collapsed to make more detailed statements.

The first step in the analysis is to rule out the homogeneity model in which all probabilities are the same ( .25 , since they are conditional on each row's stimulus combination and there are four categories/row) ${ }^{4}$ This model requires $8 d f, 1$ per stimulus combination, out of the 32 available to reflect the experimentally induced constraints. In the present case, the value of the likelihood chi-square test statistic was $2,068.55$. We will symbolize this as $G_{24}^{2}$, the subscript denoting the degrees of freedom. ${ }^{5}$ This is highly significant $(p<.001)$, rejecting the homogeneity model.

We next constrain the four expected response frequencies to match the observed frequencies of responding "word" and "nonword" to $A$, which were 2,250 ( $p=$ $56 \%)$ and $1,750(p=44 \%)$, respectively, by setting the "word"-" word" and "word"-" "nonword" proportions to $.28(.56 / 2)$ and the "nonword" "-"word" and "nonword"-" "nonword"' proportions to $.22(.44 / 2)$, respectively. The residual $G_{23}^{2}$ of $2,005.89$ is also highly significant, denoting the presence of other effects.

The difference between $G_{24}^{2}$ and $G_{23}^{2}(62.66=$ $2,068.55-2,005.89$ ) is a chi-square test statistic (it is actually more robust than the two values on which it is based; cf. Agresti \& Yang, 1986) for the $a$ bias and is highly significant $(p<.01)$. We will use the symbol $G^{2}$ (without subscripts, since all such terms in this paper involve $1 d f$ ) to denote test statistics for individual effectsthat is, effect $G^{2}$ values.

There are various ways to describe effect magnitudes. We use the estimated log-odds ratio or logit for an effect at its time of entry into the model (.13 in the present case). This index is normally available as standard output. We will use the symbol $\beta$ to denote its functional similarity to beta weights of ordinary (continuous variable) multiple regression and analysis of variance effects (another common symbol is $\lambda$ ). It is not identical to a beta weight, since it only adjusts for previous and not later terms in the model, and its metric properties are slightly different. Other measures are possible (Wickens, 1989;
Wickens \& Olzak, 1989). However, because all effects of interest were also apparent in the observed percentages after appropriate collapsing, we will also present the observed percentages.

Next, the (weaker) $b$ bias ("word" $=2,058$, $p=51 \%$, and "nonword" $=1,942, p=49 \%$ ) are constrained, producing a residual $G_{22}^{2}$ of $2,002.52$. The effect $G^{2}$ was a nonsignificant $3.37(\beta=.03)$. The overall same bias is then entered, producing a residual $G_{23}^{2}$ of $1,981.79$ and an effect $G^{2}$ of $20.73(p<.01)$ and $\beta$ of .08. This completes the adjustment for overall (marginal) biases. Table 2 contains the $\beta$ and effect $G^{2}$ values for these and subsequent effects in the first two columns of data.

The next constraint is the contingency between $a$ and its proper stimulus, $A$. Because the accuracy of $a$ was $78 \%$, this contingency is very large $\left(\beta=.64, G^{2}=\right.$ $1,317.96, p<.01)$. The accuracy of $b$ is lower $(67 \%)$, but its contingency is also large $\left(\beta=.37, G^{2}=498.90\right.$, $p<.01)$.

Were independence to hold, the residuals (observed predicted response probabilities) would be nonsignificant. This is not the case $\left(G_{19}^{2}=164.93, p<.01\right)$. Table 3

Table 2

Hierarchical Model Weights $(\beta)$ and Chi-Square Values $\left(G^{2}\right)$ for Simultaneous/Masked and Sequential/Unmasked Tasks

\begin{tabular}{|c|c|c|c|c|c|}
\hline \multirow[b]{3}{*}{ Stimulus } & \multirow[b]{3}{*}{ Response } & \multicolumn{4}{|c|}{ Experiment } \\
\hline & & \multicolumn{2}{|c|}{$\begin{array}{c}\text { Simultaneous/ } \\
\text { Masked }\end{array}$} & \multicolumn{2}{|c|}{$\begin{array}{l}\text { Sequential/ } \\
\text { Unmasked }\end{array}$} \\
\hline & & $\beta$ & $G^{2}$ & $\beta$ & $G^{2}$ \\
\hline & $a$ & .13 & $62.66 \dagger$ & .20 & $158.66 \dagger$ \\
\hline & $b$ & .03 & 3.37 & .06 & $12.55 \dagger$ \\
\hline & same & .07 & $20.73 \dagger$ & -.10 & $35.17 \dagger$ \\
\hline$A$ & $a$ & .64 & $1,317.96 \dagger$ & 1.03 & $2,398.83 \dagger$ \\
\hline$B$ & $b$ & .37 & $498.90 \dagger$ & 1.32 & $3,511.23 \dagger$ \\
\hline$A$ & $b$ & .03 & 1.73 & .02 & 0.21 \\
\hline$B$ & $a$ & .04 & 3.64 & -.09 & $4.03^{*}$ \\
\hline$A$ & same & .04 & 3.53 & .05 & 2.49 \\
\hline$B$ & same & .03 & 3.61 & .03 & 1.00 \\
\hline $\mathbf{R}$ & $a$ & .06 & $10.91 \dagger$ & .03 & 1.86 \\
\hline $\mathbf{R}$ & $b$ & .12 & $47.17 \dagger$ & .29 & $77.26 \dagger$ \\
\hline $\mathbf{R}$ & same & .05 & $8.49 \dagger$ & .00 & 0.04 \\
\hline$A \times \mathrm{R}$ & $a$ & -.02 & 0.58 & -.10 & $10.25 \dagger$ \\
\hline$B \times \mathbf{R}$ & $b$ & .05 & $7.64 \dagger$ & -.04 & 0.19 \\
\hline$A \times \mathrm{R}$ & $b$ & -.01 & 0.11 & .15 & $14.37 \dagger$ \\
\hline$B \times R$ & $a$ & .01 & 0.48 & .02 & 0.14 \\
\hline$A \times \mathrm{R}$ & same & -.02 & 1.57 & -.05 & 2.71 \\
\hline$B \times R$ & same & -.04 & $6.45^{*}$ & .02 & 0.27 \\
\hline$A \times B$ & $a$ & .08 & $13.26 \dagger$ & .16 & $9.45 \dagger$ \\
\hline$A \times B$ & $b$ & .07 & $11.28 \dagger$ & .15 & $6.48 *$ \\
\hline$A \times B$ & same & -.06 & $7.03 \dagger$ & .12 & $4.30^{*}$ \\
\hline$A \times B \times \mathrm{R}$ & $a$ & -.08 & $13.46 \dagger$ & -.05 & 0.69 \\
\hline$A \times \mathbf{B} \times \mathbf{R}$ & $b$ & -.10 & $23.60 \dagger$ & .02 & 0.21 \\
\hline$A \times B \times \mathrm{R}$ & same & .01 & 0.39 & .05 & 0.57 \\
\hline
\end{tabular}

Note-The residual $G^{2}$ values for the homogeneity model (equal expected cell frequencies) are 2,068.55 and 6,252.96, respectively, which are also the sums of the $G^{2}$ values. $R=$ Semantic relatedness. The residual term became nonsignificant after accounting for the effect of $A \times B \times \mathrm{R}$ on $b$ in the simultaneous/masked analysis and $A \times B$ on $b$ in the successive/unmasked analysis, even though the next effect in the latter case, that of $A \times B$ on same responses, was also significant. $* p<.05 .+p<.01$. $(d f=1$ in all cases. $)$ 
Table 3

Deviations (Observed - Expected) from an Independence Model (Correct Response Pairings in Italics)

\begin{tabular}{|c|c|c|c|c|c|c|c|c|}
\hline \multirow[b]{3}{*}{ Stimulus } & \multicolumn{8}{|c|}{ Experiment } \\
\hline & \multicolumn{4}{|c|}{ Simultaneous/Masked } & \multicolumn{4}{|c|}{ Sequential/Unmasked } \\
\hline & "WW" & "WN"' & "NW"' & "NN" & "WW'" & "WN"' & "NW" & "NN" \\
\hline RWW & .08 & -.06 & -.00 & -.02 & .04 & -.04 & .01 & .00 \\
\hline RWN & .07 & -.06 & .00 & -.01 & .04 & -.05 & .02 & -.00 \\
\hline RNW & .06 & -.01 & .01 & -.06 & -.01 & .00 & .01 & -.00 \\
\hline RNN & -.01 & .01 & -.00 & -.01 & .03 & .03 & .02 & -.07 \\
\hline UWW & .01 & .04 & -.01 & -.03 & .00 & .03 & -.03 & .00 \\
\hline UWN & -.11 & .04 & .00 & .07 & -.06 & .05 & -.00 & .01 \\
\hline UNW & -.10 & .02 & -.04 & .12 & -.04 & -.00 & .02 & .02 \\
\hline UNN & -.00 & .02 & .04 & -.06 & .00 & -.01 & -.04 & .05 \\
\hline
\end{tabular}

Note-The first letter denotes level of semantic relatedness $(R=$ related and $U=$ unrelated); the second letter denotes level of $A(\mathrm{~W}=$ word and $N=$ nonword $)$; and the third letter denotes level of $B(\mathrm{~W}=$ word and $\mathrm{N}=$ nonword $)$.

contains the residuals, which range from -.11 to .12 , but form no simple pattern. The further stages of the analysis help specify this pattern. ${ }^{6}$

Table 2 indicates that adjusting for the cross-contingencies of $A$ on $b$ and $B$ on $a$ has very little effect. In both cases, "word" responses are slightly but nonsignificantly more likely when the improper stimulus is also a word. Similarly, neither $A$ nor $B$ affects the probability of a same response.

Of more central interest are the contingent biases induced by relatedness. Responses to $A$ are more likely to be "word" when $A$ and $B$ are related (58\%) rather than unrelated $\left(54 \% ; \beta=.06, G^{2}=10.91, p<.01\right)$. This effect is roughly the same magnitude when $B$ is a word (61\% vs. $56 \%)$ than when it is a nonword $(56 \%$ vs. $53 \%)$. The difference is even stronger for $b(57 \%$ vs. $46 \%$; $\left.\beta=.12, G^{2}=47.17, p<.01\right)$ and holds both when $A$ was a word $(61 \%$ vs. $47 \%)$ and when it was a nonword ( $52 \%$ vs. $45 \%$ ). Relatedness also increases the probability of a same response $\left(56 \%\right.$ vs. $51 \% ; \beta=.05, G^{2}=$ $8.49, p<.01$ ). The difference is greater when $B$ is a nonword $(7 \%)$ than when $B$ is a word $(3 \% ; \beta=-.04$, $\left.G^{2}=6.45, p<.05\right)$.

The accuracy of $a$ is $78 \%$ when $A$ and $B$ are related and $77 \%$ when they are unrelated $\left(\beta=-.02, G^{2}<1\right.$, n.s.). However, $b$ is slightly more accurate when $A$ and $B$ are related $(69 \%)$ than when they are unrelated $(65 \% ; \beta=.05$, $\left.G^{2}=7.64, p<.01\right)$. There is therefore no evidence that the priming effect on $a$ involves a perceptual change in the sense of reflecting more accurate judgments, above and beyond any changes in response bias, but there is some slight evidence that the priming effect on $b$ does.

The residual $G_{6}^{2}$ at this point is $69.02(p<.01)$, and five effects involving whether $A$ and $B$ belong to the same lexical class or different lexical classes are significant in Table 2 . The residuals indicate a common reason for these effects. No residual involving related stimuli or different responses ("word"-" "nonword" and "nonword"'-"word") to unrelated stimuli exceeds \pm .01 . However, "word"-"word" responses are underpredicted by .04 with unrelated stimuli from the same lexical class (word-word or nonword- nonword) and overpredicted by this same amount with unrelated stimuli from different lexical classes (word- nonword or nonword-word). In contrast, " "nonword"-" "nonword" responses are overpredicted by .05 for stimuli in the same lexical class and underpredicted by .05 for stimuli in different lexical classes (see Table 4).

Individual differences. Any contingency analysis assumes independence of the individual observations, which, in the present case, can only be achieved if 4,000 subjects are each run for a single trial. Consequently, Table 1 reflects both between- and within-subjects effects. One possible approach to this problem is to analyze subjects separately and add their corresponding $G^{2}$ values. However, because the 80 observations/subject do not allow adequate estimation, we estimated parameters from five randomly grouped blocks of 10 subjects. Table 5 contains these estimates ranked in increasing order, the standard deviation over individuals estimated from the central limit theorem (the standard deviations of the five estimates times $10^{.5}$ because there are 10 subjects/block), and the sum of the five $G^{2}$ values, denoted $\Sigma G^{2}(d f=5)$. The means of the five estimates are omitted, since they are nearly the same as the single estimates of Table 2 .

As can be seen, nearly all effects that were significant when individual differences were ignored are also significant here. One significant effect at the group level just misses significance here $(A \times B$ on same $)$ and one significant effect was previously nonsignificant $(A \times$ relatedness on $b$ ), which is basically an artifact of one subject block.

The estimated individual subjects' standard deviations indicate that some effects vary little across subjects, most clearly the $a$ detection ( $S D=.02$ ), the $a$ marginal bias $(S D=.04)$, and, most crucially, the contingent bias of relatedness on $b(S D=.04)$. At the other extreme, the relatedness on $a$ contingent bias varies very widely $(S D=.20)$; one estimate (.19) actually was larger than any of the corresponding estimates for $b$. The perceptual priming interaction $B \times$ relatedness on $b(S D=.10)$ and 
Table 4

Deviations (Observed - Expected) Following Adjustment for Main Effects and First-Order Interactions Involving Semantic Relatedness (Correct Response Pairings in Italics)

\begin{tabular}{|c|c|c|c|c|c|c|c|c|}
\hline \multirow[b]{3}{*}{ Stimulus } & \multicolumn{8}{|c|}{ Experiment } \\
\hline & \multicolumn{4}{|c|}{ Simultaneous/Masked } & \multicolumn{4}{|c|}{ Sequential/Unmasked } \\
\hline & "WW" & "WN" & "NW" & "NN" & "WW" & "WN" & "NW" & "NN" \\
\hline RWW & -.01 & .01 & .00 & .00 & .02 & -.01 & -.01 & .00 \\
\hline RWN & .01 & -.01 & -.00 & -.00 & -.02 & .01 & .01 & -.00 \\
\hline RNW & .01 & -.01 & -.00 & -.00 & -.02 & .01 & .01 & -.00 \\
\hline RNN & -.01 & .01 & .00 & .00 & .02 & -.01 & -.01 & .00 \\
\hline UWW & .04 & .00 & .01 & -.05 & .01 & -.00 & -.00 & -.00 \\
\hline UWN & -.04 & -.00 & -.01 & .05 & -.01 & .00 & .00 & .00 \\
\hline UNW & -.04 & -.00 & -.01 & .05 & -.01 & .00 & .00 & .00 \\
\hline UNN & .04 & .00 & .01 & -.05 & .01 & -.00 & -.00 & -.00 \\
\hline
\end{tabular}

Note-The first letter denotes level of semantic relatedness $(R=$ related and $U=$ unrelated); the second letter denotes level of $A(\mathrm{~W}=$ word and $\mathrm{N}=$ nonword $)$; and the third letter denotes level of $B(\mathrm{~W}=$ word and $\mathrm{N}=$ nonword $)$.

the various first- and second-order interactions involving $A$ and $B$ are intermediate in variability.

Control versus experimental trials. We next compare control trials with $A$ and $B$ in separate analyses. Preliminary analysis revealed that subjects never mistook the " *****" for either a word or a nonword in either control condition, so we will concentrate on the event generating the lexical decision. The successive effects tested are: (1) word versus nonword stimuli; (2) experimental versus control conditions; (3) whether the other experimental stimulus was a word or a nonword; (4) cued versus uncued control conditions; (5) the interaction of (1) and (2); (6) the interaction of (1) and (3); and (7) the interaction of (1) and (4). In addition to the $\beta$ weights and associated $G^{2}$ values for each of these effects, there is also an intercept term that indexes overall response bias, since it reflects the marginal probabilities of responding "word" versus "nonword" (for further details, consult SAS In-

Table 5

Values of $\boldsymbol{\beta}$ Estimated by Blocks of 10 Subjects (Ranked), Standard Deviations $(S D)$ of Individual Estimates, and Sum of Individual $G^{2}$ Values $\left(\Sigma G^{2}\right)$ : Simultaneous/Masked Task

\begin{tabular}{|c|c|c|c|c|c|c|c|c|}
\hline \multirow[b]{2}{*}{ Stimulus } & \multirow[b]{2}{*}{ Response } & \multicolumn{5}{|c|}{ Block } & \multirow[b]{2}{*}{$S D$} & \multirow[b]{2}{*}{$\Sigma G^{2}$} \\
\hline & & 1 & 2 & 3 & 4 & 5 & & \\
\hline & $a$ & .10 & .11 & .12 & .13 & .14 & .04 & $57.01 \dagger$ \\
\hline & $b$ & -.02 & .01 & .03 & .04 & .05 & .06 & 4.40 \\
\hline & same & .05 & .06 & .07 & .10 & .11 & .06 & $27.40 \dagger$ \\
\hline$A$ & $a$ & .63 & .63 & .64 & .64 & .65 & .02 & $1,287.97 \dagger$ \\
\hline$B$ & $b$ & .31 & .35 & .37 & .38 & .40 & .07 & $498.01 \dagger$ \\
\hline$A$ & $b$ & .00 & .01 & .02 & .04 & .04 & .04 & 2.03 \\
\hline$B$ & $a$ & -.01 & .00 & .04 & .04 & .11 & .11 & 7.25 \\
\hline$A$ & same & .00 & .04 & .05 & .05 & .06 & .05 & 4.98 \\
\hline$B$ & same & .01 & .02 & .03 & .04 & .08 & .06 & 6.04 \\
\hline $\mathbf{R}$ & $a$ & -.02 & -.01 & .04 & .12 & .19 & .20 & $27.91 \dagger$ \\
\hline $\mathbf{R}$ & $b$ & .09 & .09 & .12 & .13 & .15 & .06 & $46.54 \dagger$ \\
\hline $\mathbf{R}$ & same & .01 & .02 & .04 & .09 & .09 & .08 & $12.72^{*}$ \\
\hline$A \times \mathrm{R}$ & $a$ & -.09 & -.04 & -.02 & .02 & .03 & .11 & 5.48 \\
\hline$B \times \mathrm{R}$ & $b$ & .01 & .02 & .03 & .09 & .10 & .10 & $12.85^{*}$ \\
\hline$A \times \mathrm{R}$ & $b$ & -.14 & -.03 & .03 & .04 & .04 & .18 & $11.98^{*}$ \\
\hline$B \times \mathbf{R}$ & $a$ & -.04 & .01 & .03 & .03 & .04 & .07 & 2.38 \\
\hline$A \times \mathbf{R}$ & same & -.12 & -.03 & -.02 & .01 & .02 & .13 & 7.27 \\
\hline$B \times \mathrm{R}$ & same & -.11 & -.06 & -.05 & -.03 & .02 & .10 & $12.14 *$ \\
\hline$A \times B$ & $a$ & .04 & .05 & .09 & .11 & .11 & .07 & $15.61 \dagger$ \\
\hline$A \times B$ & $b$ & -.00 & -.00 & .07 & .11 & .18 & .17 & $2.64 \dagger$ \\
\hline$A \times B$ & same & -.16 & -.08 & -.04 & -.04 & -.02 & .13 & 1.95 \\
\hline$A \times B \times \mathrm{R}$ & $a$ & -.15 & -.11 & -.11 & -.05 & .02 & .15 & $17.58 \dagger$ \\
\hline$A \times B \times \mathrm{R}$ & $b$ & -.17 & -.15 & -.11 & -.08 & .01 & .16 & $25.96 \dagger$ \\
\hline$A \times B \times R$ & same & .00 & .00 & .01 & .01 & .02 & .00 & .26 \\
\hline
\end{tabular}

Note-The values of $\beta$ obtained by blocks have been ranked in ascending order. The value of $S D$ is the standard deviation of these five values of $\beta$ times $\sqrt{ } 10$ (the number of subjects per block), so that $S D$ estimates variability of effect magnitude over individuals instead of blocks. Finally, $\Sigma G^{2}$ is the sum of the individual $G^{2}$ values corresponding to the five values of $\beta$. $\mathrm{R}=$ semantic relatedness. ${ }^{*} p<.05 . \dagger p<.01$. 
stitute, 1985b, or Wickens, 1989). The overall purpose of the analysis is to evaluate the effects of making one versus two decisions.

The previously considered Effects 3 and 6 are only included to provide proper model adjustments and deal exclusively with experimental condition performance. The intercept and Effect 1 pool across the experimental and control conditions and thus are not of interest. This leaves Effects 2, 4, 5, and 7 to discuss.

There is no difference in bias between control and $A$ trials. "Word" responses occurred 55\% of the time in both cases $\left(\beta=.04, G^{2}=2.26\right.$, n.s.). The subjects responded "word" on 54\% of the cued control trials versus $46 \%$ of the uncued trials. This difference is not significant $\left(\beta=.04, G^{2}=1.01\right)$. However, the subjects were slightly more accurate on control (83\%) trials than on experimental trials $\left(78 \% ; \beta=.16, G^{2}=16.00\right.$, $p<.01)$. Finally, the subjects were more accurate on cued control trials $(88 \%)$ than on uncued control trials $\left(67 \% ; \beta=-.38, G^{2}=73.23, p<.01\right)$.

The subjects were more likely to say "word" on control trials (54\%) than on $B$ trials $\left(\beta=-.09, G^{2}=13.01\right.$, $p<.01$ ), and they were more accurate on control trials $(83 \%)$ than on $B$ trials $\left(67 \% ; \beta=-.44, G^{2}=272.53\right.$, $p<.01)$. The remaining two effects were considered in the preceding paragraph.

\section{Discussion}

In sum, the major deviation from independence is that relatedness induces a contingent bias primarily affecting the right-hand stimulus. Given the left/right order of both reading and response, we consider the left event to be processed first and the right event to be processed second. The proactive bias is very consistent across subjects and depends little on the first event's lexical class. It supports Farah's (1989) conclusion that relatedness causes a criterion shift in favor of "word" responses.

\section{EXPERIMENT 2}

The Experiment 2 configuration, called sequential/unmasked, involves presenting the stimuli successively to the same retinal location; masks are not used.

\section{Method}

Subjects. Fifty introductory psychology students met the same requirements as in Experiment 1.

Stimuli and Apparatus. Two of the three stimulus lists from Experiment 1 (experimental and cued control) were employed. The apparatus was identical to that used previously.

Procedure. As in Experiment 1, the subjects were led into the laboratory, given a brief description of the experimental procedure, and dark-adapted for $5 \mathrm{~min}$. They were then presented with $80 \mathrm{ex}$ perimental and 40 cued control trials as in Experiment 1; uncued control trials were omitted in the interest of time. Conditions and trials within conditions were again randomized, and the subjects were again given 4 practice trials/condition.

Experimental trials. On each experimental trial, subjects were exposed to: (1) the 900 -msec fixation cross, (2) a 500-msec blank screen, (3) $A$ for $250 \mathrm{msec}$, and, finally, (4) $B$ for $250 \mathrm{msec}$. As before, no subject's performance was perfect. Both stimuli were centered at fixation. The subjects made lexical decisions in the order of stimulus appearance.

Control trials. Control trials paralleled Experiment l's cued control condition, except that the fixation event also cued either the first event ("F") or the second event ("S").

\section{Results}

Table 1 also contains the data from the sequential/ unmasked task. The unconditional biases for $a$ and $b$ are slightly stronger than those obtained in Experiment 1 $[p$ ("word") $=.60$ for $a$ and .53 for $b$ ]. Unlike in Experiment 1 , there is a marginal bias toward responding different $\left(\beta=-.10, G^{2}=35.17, p<.01\right)$. Accuracies are higher ( $a=85 \%$ and $b=93 \%$ ) because the presentation time was the same as in Experiment 1, but the stimuli were not masked. Responses to $B$ were more accurate than responses to $A$ because $B$ was a strong backward mask for $A$, but $A$ was only a weak forward mask for $B$.

Table 3 indicates the residuals beyond independence. As in Experiment 1, there is no cross contingency of $A$ on $b$, but there is a modest inhibitory effect of $B$ on $a$. The $a$ responses are "word" $64 \%$ of the time when $B$ is a word versus $69 \%$ when $B$ is a nonword $(\beta=-.09$, $G^{2}=4.03, p<.05$ ).

The bias in $b$ toward "word" with related versus unrelated pairings is similar in magnitude to that in Experiment 1 , despite the greater overall accuracy here $(57 \%$ vs. $\left.47 \% ; \beta=.29, G^{2}=77.26\right)$. Although $A$ 's lexical class affects the magnitude of the difference $(\beta=.15$, $G^{2}=14.37$ ), it does not affect the order. The "word" percentages for $b$ are $56 \%$ versus $47 \%$ when $A$ is a word and $57 \%$ and $52 \%$ when $A$ is a nonword. The effect of relatedness on "word" responses to $a$ is minimal ( $61 \%$ vs. $59 \% ; \beta=.03, G^{2}=1.86$, n.s.).

Unlike in Experiment 1, relatedness affects $a$ 's accuracy, but the effect is inhibitory $\left(\beta=-.10, G^{2}=\right.$ $10.25, p<.01 ; 84 \%$ vs. $87 \%$ for related and unrelated pairings). Relatedness leads to an identical 3\% nonsignificant decrease in $b$ 's accuracy $(91 \%$ vs. $94 \% ; \beta=-.04$, $G^{2}=.14$ ). The difference in $a$ and $b$ bias is responsible for the different statistical outcomes.

Both $a$ and $b$ are more accurate when the improper stimulus is a word rather than a nonword $(89 \%$ vs. $82 \%$ and $94 \%$ vs. $92 \% ; \beta=.16$ and $.15, G^{2}=9.45$ and 6.48 ). In addition, when $A$ is a word, same responses are more likely on related than on unrelated trials $(54 \%$ vs. $.48 \%$ ) but when $A$ is a nonword, the reverse is true (39\% vs. $44 \% ; \beta=.12, G^{2}=4.30$ ). The complex interaction found in Experiment 1 (Table 4) was not found and no residual exceeded \pm .03 following adjustment for the $A \times$ relatedness effect on $b$.

Individual differences. Table 6 parallels Table 5 in providing estimates by subject blocks. Four effects that were significant in the overall analysis (Table 2 ) miss significance here: (1) the cross contingency of $B$ on $a$, (2) the $A \times$ relatedness interaction on $a$, (3) the $A \times B$ interaction on $b$, and (4) the $A \times B$ interaction on same. The effect of relatedness on $b$ remains at roughly the same level of 
Table 6

Values of $\beta$ Estimated by Blocks of 10 Subjects (Ranked), Standard Deviations (SD) of Individual Estimates, and Sum of Individual $G^{2}$ Values $\left(\Sigma G^{2}\right)$ : Sequential/Unmasked Task

\begin{tabular}{|c|c|c|c|c|c|c|c|c|}
\hline \multirow[b]{2}{*}{ Stimulus } & \multirow[b]{2}{*}{ Response } & \multicolumn{5}{|c|}{ Block } & \multirow[b]{2}{*}{$S D$} & \multirow[b]{2}{*}{$\Sigma G^{2}$} \\
\hline & & 1 & 2 & 3 & 4 & 5 & & \\
\hline & $a$ & .02 & .10 & .13 & .18 & .21 & .16 & $81.16 \dagger$ \\
\hline & $b$ & -.11 & -.06 & -.02 & .01 & .05 & .14 & $14.07 *$ \\
\hline & same & -.18 & -.15 & -.11 & -.08 & -.02 & .14 & $52.01 \dagger$ \\
\hline$A$ & $a$ & .86 & .91 & .94 & 1.03 & 1.05 & .17 & $1,904.59 \dagger$ \\
\hline$B$ & $b$ & 1.18 & 1.24 & 1.28 & 1.33 & 1.34 & .15 & $3,105.85 \dagger$ \\
\hline$A$ & $b$ & -.09 & -.08 & .05 & .05 & .07 & .17 & 3.51 \\
\hline$B$ & $a$ & -.18 & -.16 & -.09 & -.03 & .07 & .22 & 6.53 \\
\hline$A$ & same & -.01 & .03 & .05 & .07 & .11 & .09 & 4.66 \\
\hline$B$ & same & -.08 & -.02 & -.00 & .08 & .10 & .17 & 4.30 \\
\hline $\mathbf{R}$ & $a$ & -.00 & .02 & .02 & .06 & .07 & .07 & 3.31 \\
\hline $\mathbf{R}$ & $b$ & .22 & .24 & .27 & .30 & .34 & .11 & $67.14 \dagger$ \\
\hline $\mathbf{R}$ & same & -.09 & -.02 & -.00 & .02 & .03 & .11 & 3.98 \\
\hline$A \times \mathrm{R}$ & $a$ & -.13 & -.12 & -.10 & -.06 & -.05 & .08 & 8.99 \\
\hline$B \times \mathrm{R}$ & $b$ & -.14 & -.06 & -.05 & -.04 & .01 & .12 & 3.13 \\
\hline$A \times \mathrm{R}$ & $b$ & -.19 & -.13 & -.04 & .01 & .04 & .21 & 6.59 \\
\hline$B \times \mathrm{R}$ & $a$ & -.10 & .08 & .18 & .27 & .44 & .46 & $22.29 \dagger$ \\
\hline$A \times \mathrm{R}$ & same & -.15 & -.13 & -.09 & -.02 & .02 & .17 & 7.42 \\
\hline$B \times \mathrm{R}$ & same & -.15 & .01 & .01 & .04 & .08 & .20 & 3.58 \\
\hline$A \times B$ & $a$ & .00 & .11 & .14 & .32 & .33 & .23 & $15.33 \dagger$ \\
\hline$A \times B$ & $b$ & -.04 & -.03 & .03 & .17 & .21 & .25 & 2.96 \\
\hline$A \times B$ & same & .00 & .00 & .00 & .00 & .45 & .00 & 8.40 \\
\hline$A \times B \times \mathrm{R}$ & $a$ & -.01 & .00 & .00 & .00 & .00 & .00 & .02 \\
\hline$A \times B \times \mathrm{R}$ & $b$ & -.10 & .00 & .00 & .00 & .16 & .00 & 1.94 \\
\hline$A \times B \times \mathrm{R}$ & same & .00 & .00 & .00 & .00 & .00 & .00 & .00 \\
\hline
\end{tabular}

Note-The values of $\beta$ obtained by blocks have been ranked in ascending order. The value of $S D$ is the standard deviation of these five values of $\beta$ times $\sqrt{ } 10$ (the number of subjects per block), so that $S D$ estimates variability of effect magnitude over individuals instead of blocks. Finally, $\Sigma G^{2}$ is the sum of the individual $G^{2}$ values corresponding to the five values of $\beta$. $\mathrm{R}=$ semantic relatedness. ${ }^{*} p<.05 . \dagger p<.01$.

consistency as in Experiment $1(S D=.11)$. The nonsignificant effect of relatedness on $a$ is even more consistent $(S D=.07)$. Finally, the $B \times$ relatedness interaction on $a$, which is significant here but not in the overall analysis, varies very widely across subjects $(S D=.46)$.

Control versus experimental trials. Since we only obtained cued control data, the only effects of interest are the relative bias and accuracy in control and experimental trials, separately for $a$ and $b$ experimental trials.

The subjects had a stronger "word" bias for $a$ on experimental than on control trials $(60 \%$ vs. $55 \% ; \beta=.16$, $G^{2}=30.21, p<.01$ ), and they were also less accurate ( $85 \%$ vs. $\left.92 \% ; \beta=-.17, G^{2}=20.20, p<.01\right)$. Conversely, the subjects had a weaker "word" bias for $b$ than they did on control trials ( $53 \%$ vs. $55 \% ; \beta=-.11$, $G^{2}=10.52, p<.01$ ). There is no difference in $b$ 's accuracy and control accuracy ( $92 \%$ in both cases; $\beta=.00$, $G^{2}=2.01$, n.s.).

\section{Discussion}

The primary effect of interest is again the bias toward saying "word" to the second event with semantically related stimuli, which was extremely consistent across subjects. Relatedness may induce a slight inhibitory effect on first-event decisions (significant in the overall analysis, but just missing significance in the analysis by blocks). Finally, we noted higher order differences between stimuli in the same versus different lexical classes which are relatively variable across subjects and thus perhaps reflected strategic idiosyncracies.

\section{GENERAL DISCUSSION}

The present results indicate that relatedness induces strong proactive biases that are highly consistent across subjects. Retroactive bias effects are variable across subjects in Experiment 1 and nonsignificant in Experiment 2. Accuracy differences are nonsignificant and/or inconsistent in direction. Performance never exceeds parity.

Stone and Van Orden (1989), whose study appeared after our data were gathered, provide the only other study we know in which retroactive and proactive effects are directly compared. Stone and Van Orden's accuracy task differs from ours in two ways. First, subjects made only one response per trial, with proactive and retroactive primes surrounding the discriminanda in time. Second, Stone and Van Orden used what they termed "legal" primes (misspellings) and "illegal" primes (anagrams of related words). Word primes induced slightly greater proactive effects than retroactive effects. Nonwords of both types failed to produce priming; error scores were, if anything, lower with unrelated nonword primes. They 
note that their results differed from Briand et al. (1988), who found backward priming with "legal" nonword foils to asynchronies of up to $1 \mathrm{sec}$.

Our nonwords were equivalent to Stone and Van Orden's (1989) "illegal" nonwords, for they too were anagrams, but they were as effective as words in inducing priming. However, their primes were much more intense than their targets, being presented in upper- as opposed to lowercase, in larger type, and for longer durations $(1.5 \mathrm{sec})$ than the target. More intense stimuli should provide better discrimination between words and nonwords. Consequently, the absence of "wordlikeness" would be more apparent in their study than ours. If a word and its nonword anagram are imperfectly discriminable from each other, as we intended them to be, it is not surprising that the effects of the two would be similar. Consequently, this disparity in our results and Stone and Van Orden's seems readily understandable.

The present results support Farah's (1989) criterion shift hypothesis and Schvaneveldt and McDonald's (1981) accuracy data. However, Bernstein et al. (1989) found accuracy facilitation previously, as did Briand et al. (1988). The present task seemed a most straightforward way to create double judgments that parallel single judgments. One can readily see how each stimulus could make the other appear more "wordlike" and that the task did not provide the equivalent of Schvaneveldt and McDonald's (1981) "second look." Bernstein et al.'s (1989) tasks (identification and recognition) were much more amenable to "second looks" to restrict the possible response alternatives. They noted that seeing "TEXAS CITY" clearly and another word with "LL" in the middle could make "DALLAS" more probable than "DULLES." The same prime here would not help one discriminate between "DALLAS" and "DALLSA."

It seems most curious why these biases were so clearly asymmetrical when the task had the potential to produce symmetry. Nothing prevented the subject from responding via a Ratcliff and McKoon (1988) type compound (which was originally intended to deal with recall and not lexical decisions ${ }^{7}$ ), since reactions were untimed. We did find slight evidence for compounding in the higher order interactions. However, the stronger second-event bias produced by relatedness was found regardless of which discrimination was easier.

Perhaps the most provocative explanation of priming is Neely's (1977) semantic activation. It very clearly predicts our stronger proactive effects, although it could be formulated to allow weaker retroactive effects. It readily accounts for biases, because it explicitly assumes that related nonwords are activated along with related words. Higher activations lead, in turn, to more "word" responses. Subsidiary assumptions about the relative activation of words and nonwords are needed to account for accuracy changes.

Although these data are in accord with a spreading activation model, our previous results (Bernstein et al., 1989) are not; the reverse is true regarding Ratcliff and McKoon's (1988) compound cue model. The present studies show proactive response biases with minimal retroactive effects. Our earlier study showed strong retroactive and apparently perceptual effects (ordinary proactive priming was not assessed). Neither set of results was totally unprecedented. Consequently, processes like Schvaneveldt and McDonald's (1981) "second look," Neely's (in press) semantic matching, or McClelland and Rumelhart's (1981) interactive activation seem needed to go beyond pure spreading activation effects. "Second looks" are an especially attractive possibility, because they can affect perceptual stages. As desirable as it would be to have all priming operate in the parsimonious manner suggested by Farah (1989), this does not seem possible, given the diversity of results.

\section{REFERENCES}

Agresti, A., \& Y ANG, M. (1986). An empirical investigation of some effects of sparseness in contingency tables. Computational Statistics \& Data Analysis, 5, 9-21.

Antos, S. J. (1979). Processing facilitation in a lexical decision task. Journal of Experimental Psychology: Human Perception \& Performance, 5, 527-545.

Ashby, F. G., \& Townsend, J. T. (1986). Varieties of perceptual independence. Psychological Review, 93, 154-179.

Battig, W. F., \&ontague, W. E. (1969). Category norms for verbal items in 56 categories: A replication and extension of the Connecticut category norms. Journal of Experimental Psychology Monographs, 80.

Bernstein, I. H., Bissonnette, V., Vyas, A., Barclay, P. (1989). Semantic priming: Subliminal perception or context? Perception \& Psychophysics, 45, 153-161.

Bishop, Y. M. M., FienberG, S. E., \& Holland, P. W. (1975). Discrete multivariate analysis: Theory and practice. Cambridge, MA: MIT Press.

Briand, K., den Heyer, K. \& Dannebring, G. L. (1988). Retroactive semantic priming in a lexical decision task. Quarterly Journal of Experimental Psychology, 40A, 341-359.

Carr, T. H., McCauley, C., Sperber, R. D., \& Parmelee, C. M. (1982). Words, pictures, and priming: On semantic activation, conscious identification, and the automaticity of information processing. Journal of Experimental Psychology: Human Perception \& Performance, 8, 757-777.

Diener, D., \& SMEe, W. P. (1984). Apple tachistoscope. Behavior Research Methods, Instruments, \& Computers, 16, 540-544

FARAH, M. J. (1989). Semantic and perceptual priming: How similar are the underlying mechanisms? Joumal of Experimental Psychology: Human Perception \& Performance, 15, 188-194.

GARNER, W. R., HAKE, H. W., ERIKSEN, C. W. (1956). Operationism and the concept of perception. Psychological Review, 63, 149-159.

Garner, W. R., \& MORTON, J. (1969). Perceptual independence: Definitions, models, and experimental paradigms. Psychological Bulletin, 72, 233-259.

Hines, D., Czerwinski, M., Sawyer, P. K., \& DWyer, M. (1986). Automatic semantic priming: Effect of category level and word association level. Journal of Experimental Psychology: Human Perception \& Performance, 12, 370-379.

LUPKER, S. J., \& MASSARo, D. W. (1979). Selective perception without confounding contributions of decision and memory. Perception \& Psychophysics, 5, 60-69.

MarCEL, A. J. (1980). Conscious and preconscious recognition of polysemous words: Locating the selective effects of prior verbal context. In R. S. Nickerson (Ed.), Attention and performance VIII (pp. 435457). Hillsdale, NJ: Erlbaum.

MarCel, A. J. (1983a). Conscious and unconscious perception: Experiments on visual masking and word recognition. Cognitive Psychology, 15, 197-237.

MARCEL, A. J. (1983b). Conscious and unconscious perception: An ap- 
proach to the relations between phenomenal experience and perceptual processes. Cognitive Psychology, 15, 238-300.

McCi.eliand, J. L., \& Rumelhart, D. E. (1981). An interactive activation model of context effects in letter perception: Part I. An account of basic findings. Psychological Review, 88, 375-407.

MFyer, D. E., Schvaneveldt, R. W. (1971). Facilitation in recognizing pairs of words: Evidence of a dependence between retrieval operations. Journal of Experimental Psychology, 90, 227-234.

Morton, J. (1969). Interaction of information in word recognition. Psychological Review, 76, 165-178.

Mül.LER, H. J., \& FINDI.AY, J. M. (1987). Sensitivity and criterion effects in the spatial cuing of visual attention. Perception \& Psychophysics, 42, 383-399.

Nrel.y, J. H. (1977). Semantic priming and retrieval from lexical memory: Roles of inhibitionless spreading activation and limited capacity attention. Journal of Experimental Psychology: General, 106. 226-254.

NeELY, J. H. (in press). Semantic priming effects in visual word recognition: A selective review of current findings and theories. In D. Besner \& G. Humphreys (Eds.), Basic processes in reading: Visual word recognition. Hillsdale, $\mathrm{NJ}$ : Erlbaum.

NorRIS, D. (1984). The mispriming effect: Evidence of an orthographic check in the lexical decision task. Memory \& Cognition, 12, 470-476.

O'CONNOR, R. E., \& ForSTER, K. I. (1981). Criterion bias and search sequence bias in word recognition. Memory \& Cognition, 9, 78-92.

Ol.zAK, L. A., \& Wickens, T. D. (1983). The interpretation of detection data through direct multivariate frequency analysis. Psychological Bulletin, 93, 574-585.

RatClifF, R., \& MCKoon, G. (1988). A retrieval theory of priming in memory. Psychological Review, 95, 385-408.

SAS INSTITUTE, INC. (1985a). SAS IML guide for personal computers. Version 6 edition. Cary, NC: Author.

SAS Institute, InC. (1985b). SAS user's guide: Statistics, Version 5 edition. Cary, NC: Author.

Schvanevel.d, R. W., MCDonald, J. E. (1981). Semantic context and the encoding of words: Evidence for two modes of stimulus analysis. Journal of Experimental Psychology: Human Perception \& Performance, 7, 673-687.

Stone, G. O., VAn Orden, G. C. (1989). Are words represented by nodes? Memory \& Cognition, 17, 511-524.

WICKENS. T. D. (1989). Multiway contingency tables analysis for the social sciences. Hillsdale, NJ: Erlbaum.
Wickens, T. D., Olzak, L. A. (1989). The statistical analysis of concurrent detection ratings. Perception \& Psychophysics, 45. 514-528.

\section{NOTES}

1. In other words, word and nonword refer to stimulus alternatives and "word" and "nonword" refer to response alternatives.

2. Wickens (1989) prefers "association" to "interaction." His point is well taken if there is no clear distinction between independent and dependent variables. However, our "interactions" are defined essentially in the same way that ANOVA interactions are defined and have the same interpretation.

3. The stimulus lists were not balanced by position, so we ran an additional group of 6 subjects in a separate study. Their performance fell within the limits of those reported in the main study.

4. The analysis can be performed by SAS PROC CATMOD (SAS Institute, 1985b), among others; we modified a SAS PROC IML program (SAS Institute, 1985a, pp. 120-124).

5. The residual likelihood chi-square is defined as $2 \Sigma f_{i} \cdot \ln \left(f_{i} / e_{i}\right)$, where $f_{i}$ is the observed frequency for the $i$ th stimulus combination, $e_{i}$ is the corresponding expected frequency $(n / 4$, where $n=$ the number of trials per stimulus combination $=50$ subjects $\times 10$ trials per subject $=500$ ), and $\ln$ is the natural $\log$ function. This form of chi-square is used rather than the more familiar Pearson statistic, $\Sigma\left(f_{i}-e_{i}\right)^{2} / e_{i}$, for statistical convenience in partitioning the table. Likelihood chi-squares are commonly denoted $G^{2}$ rather than $\chi^{2}$ to avoid confusion with Pearson's statistic.

6. There is no indisputable ordering of the remaining effects, but alternative entry orderings adhering to the hierarchical principle produced equivalent results. In general, the later an effect is entered, the more conservative the test.

7. Ratcliff (personal communication, January 5,1990 ) noted that the Ratcliff-McKoon model need not predict symmetrical results, because $b$ may be influenced by $a$ as well as the proper and improper stimulus, and a variety of strategies may influence the ultimate decision. Our point, however, is that there is nothing a priori that would prevent symmetry.

(Manuscript received January 12, 1990; revision accepted for publication June 11, 1990.) 